[0212-7199 (2004) 21: 11; pp 521-522] ANALES DE MEDICINA INTERN Copyright (C) 2004 ARAN EDICIONES, S.L.

AN. MED. INTERNA (Madrid) Vol. 21, N. ${ }^{\circ} 11$, pp. 521-522, 2004

\title{
¿Réquiem por la Medicina Interna?
}

Rico Irlés J. ¿Réquiem por la Medicina Interna? An Med Interna (Madrid) 2004; 21: 521-522.

Podemos afirmar que la Medicina Interna en el mundo occidental ha sufrido una tremenda transformación desde su nacimiento a finales del siglo XIX en Alemania. Frente a la Medicina General, que ya tenía sus elementos importantes, las grandes figuras de aquella época dieron en llamar Medicina Interna a "el conjunto de ciencias médicas aplicadas a la clínica", es decir, la profundización en el pensamiento médico, abarcando los hallazgos fisiopatológicos y anatomopatológicos y atendiendo especialmente a la visión conjunta del enfermo. O sea: el enfermo como un todo. Los nombres de Strümpell (autor del primer tratado de Medicina Interna en 1883) (1), Von Mering, L. Krehl (primer Manual de Medicina Interna en 1901) (2), deben figurar en la primera línea de la nueva disciplina. Insisto: lo más importante de este idea fue la visión conjunta del enfermo.

El término utilizado por el americano W. Osler, que calificó a los internistas como "generalistas distinguidos y plurales”, no aportó nada nuevo a la definición inicial aunque sí sus aportaciones personales a la clínica (3).

No obstante, y ya desde el principio, los creadores del término eran conscientes de la profundidad de la idea de la Medicina Interna.

Transcribo literalmente: "El campo de la Medicina Interna es tan dilatado hoy día... y es tan grande la profundidad de sus conocimientos, que resulta imposible para un solo individuo trabajar con igual acierto en todos los capítulos" (L. Krehl, 1901) (2). Es decir, que ya quedaba abierto el campo a las especialidades médicas.

Y así, y desde el principio, la Medicina Interna comenzó a desarrollar las especialidades, que salieron de ella.

No voy a enumerarlas ahora, pero de las grandes escuelas de Alemania y después de Francia, Inglaterra y posteriormente EE.UU., fueron apareciendo los creadores de las especialidades que hoy conocemos.

Y en España ocurre igual, aunque con cierto retraso y después de haber aprendido en el extranjero. Las figuras de D.
Carlos Jiménez Díaz y A. Pedro-Pons son un ejemplo clarísimo entre nosotros. Frase de éste último es: "No existe oposición entre especialidades y Medicina Interna: pues ésta es el centro desde donde irradian las especialidades" (Congreso de la Sociedad Española de Medicina Interna, 1969) (4).

Tras la muerte de éstos, y con la evolución y desarrollo de las ciencias médicas, las especialidades se han ido desarrollando más y más. Y la Medicina Interna, en su sentido primigenio, se ha ido perdiendo. Sí, digámoslo sin prejuicios ni medias palabras.

¿Qué es hoy la Medicina Interna a nivel de los grandes hospitales? El rincón donde se almacenan los enfermos con múltiples patologías. Y naturalmente, estos enfermos son muy mayoritariamente los ancianos. De este modo, la Medicina Interna es hoy una geriatría encubierta. Y la geriatría, otra especialidad más, surgida de la Medicina Interna, le disputa, y con razón, su lugar (5).

Esta es la situación actual de la Medicina Interna. Otra cosa es a nivel de los hospitales regionales, donde se mantiene el papel de la Medicina Interna, y los especialistas actúan más o menos en coordinación con ella.

Por este motivo yo me pregunto si la Medicina Interna debe dejar su lugar y desaparecer, una vez cumplido su ciclo, o debe mantenerse como movimiento coordinador de los conocimientos médicos que hoy superabundan. Y todo ello en beneficio del enfermo.

¿Es que no nos hemos dado cuenta todavía de la cantidad de fármacos que se prescriben a una sola persona procedentes del especialista cardiólogo, digestólogo, endocrinólogo, etc., y que a veces son repetición de lo mismo o a veces nocivos para otra patología que el paciente presenta? Y más aún: en los últimos tiempos han proliferado las "unidades": de hipertensión, de lípidos, de enfermedades sistémicas... No digamos ya las unidades de "enfermedades raras". Algunas de estas unidades están más que justificadas por el material que se utiliza o los datos analíticos. Otras sólo sirven para seleccionar 
los enfermos. Así, lo enfermos "interesantes" quedan en estas unidades. Los pobres viejecitos con Alzheimer, ictus residuales, EPOC, etc., pasan indefectiblemente a las salas de Medicina Interna. Y esto lleva a la desilusión y frustración de los internistas.

Como fin de estas líneas quisiera recordar que en 1994, en Wiessbaden y en la Kursaal, el mismo lugar en que tuvo lugar la primera reunión de la Sociedad Alemana de Medicina Interna un siglo antes, se celebró el centenario de la Medicina Interna (6).

Las conclusiones a que se llegó fueron sucintamente las siguientes:

1. La necesidad de ahorrar. La Medicina en general se ha hecho muy cara.

2. La necesidad de la Medicina Interna de abandonar su "torre de marfil".

3. La conveniencia de re-elaborar el concepto de Medicina Interna y de armonizar los conceptos con los demás países de la Unión Europea.

O sea, los pies en el suelo.

Sirvan estas reflexiones para que nosotros, también en España nos adaptemos a lo que la Medicina Interna nos puede seguir ofreciendo y que es fundamentalmente:

1. El sentido unitario de la Medicina y del enfermo como un todo.

2. La humildad para colaborar con las especialidades médicas que se han desarrollado tan extraordinariamente y en tantas ocasiones desbordan los límites de la madre.

Ese papel unificador y coordinador sigue siendo funda- mental en la Medicina Interna y debe ser desarrollado tanto a nivel de hospital como en la asistencia domiciliaria.

Tanto a nivel docente (en pre y postgrado y en la formación continuada), como a nivel asistencial, el servicio de Medicina Interna debe mantener un papel coordinador, fomentar las sesiones clínicas con otras especialidades (que deben ser periódicas y frecuentes); y también desde allí debe ser fomentada la investigación, si bien ésta será siempre de verificación y nunca básica o fundamental.

Si se mantienen estos criterios en nuestros hospitales y centros de salud, mirando siempre al paciente como un todo, como una persona que necesita no solo de la ciencia sino también del afecto que todo enfermo requiere $(7,8)$, sea cual sea su edad, entonces la Medicina Interna sobrevivirá a la avalancha de conocimientos. En caso contrario será absorbida y diluida cada vez más por las Especialidades que en su día nacieron de ella.

Ojalá todas las ideas aportadas por el grupo del Prof. Portugal Álvarez y cols. (9) en un atractivo proyecto técnico de gestión y funcionamiento de una unidad de Medicina Interna, sean muy pronto una clara realidad; y con ello, que estas líneas mías pudieran ser arrojadas a la papelera. Es lo que más desearía.

\section{J. RICO IRLÉS}

Servicio de Medicina Interna. Hospital Clínico Universitario San Cecilio. Facultad de Medicina. Universidad de Granada

\section{Bibliografía}

1. Strümpell A. Lehrbuch der speziellen Pathologie und Therapie der inneren Krankheiten. Leipzig 1883 (Edicion española en 1885).

2. Krehl L. Handbuch der inneren Medizin. Halle, 1901. Ed española en 1921.

3. Bean WB.Origin of the term "Internal Medicine". N Engl J Med 1982; 306 (3): 82-3.

4. Pedro-Pons A.VIII Reunión de la Sociedad Española de Med Interna. Madrid 1969. p. 13-4.

5. Editorial.Geriatrics and the limits of modern Medicine. N Engl J Med 1999; 340 (16): 1283-5.
6. Editorial.The 100th Congress of the German Society for internal Medicine.Praxis Magazin Med 1994; 5: 88-90.

7. Jovell AJ. Medicina basada en la afectividad. Med Clin (Barc) 1999; 113: 173-5.

8. Goodwin SJ. Geriatrics and the limits of the moderne Medicine. N Engl J Med 1999; 340: 1283-5.

9. Castillo Rueda, A, Portugal Álvarez J. Proyecto técnico y de gestión de funcionamiento de la unidad asistencial de Medicina Interna. An Med Interna (Madrid) 2004; 21 (1): 31-8. 